\title{
FLUOROSCOPIC EPIDURAL ANAESTHESIA: A RESCUING SKILL IN DIFFICULT SITUATION
}

\author{
Paliwal B, Bhatia P, Sharma R, Kamal M, Kumar R \\ All India Institute of Medical Sciences, Jodhpur, INDIA
}

Introduction: C-arm or fluoroscopy guided epidural injections are frequently employed in management of chronic pain conditions. We used the technique for anaesthesia and intraoperative management of a patient.

A 26 year old, male patient with polio, severe lumbar scoliosis along with deformity of the thoracic spine had to undergo urethroplasty for urethral stricture.

Breath holding time- $10 \mathrm{sec}$, Pulmonary function test- severe restrictive lung disease, Echocardiography- mild pulmonary hypertension, Cobb's angle-70 degree

Risk of cardiorespiratory events and postoperative mechanical ventilatory support with difficult weaning in case general anaesthesia is provided.

Considering the degree of spinal torsion, it was difficult to perform blind epidural and that too without avoiding patient discomfort from multiple prick attempts.

\section{Fluoroscopy guided epidural (interlaminar approach)}

1. Position: Prone/semiprone. Difficult to position supine due to khyphoscoliosis

2. Anteroposterior(AP)view: Multiple adjustments of C-arm to get true AP view without artifacts of operating table shadow

3. True anteroposterior view

4. Cranial tilt: Endplate squaring (L4-L5 interspace)

5. Metal marker at L4 lamina for needle entry point

6. Hitting lamina and walking over it till loss of bony contact

7. Loss of resistance technique to enter epidural space

8. Lateral view, dye confirmation - Vertical spread

9. Epidural catheter placement

Other approaches :

1. Transforaminal approach

2. Caudal Epidural

Limitation: Not suitable for fractures and patients in pain on movement.

\section{Conclusion:}

Knowledge of fluoroscopy technique adds to the anaesthesiologist armamentarium in difficult situations.

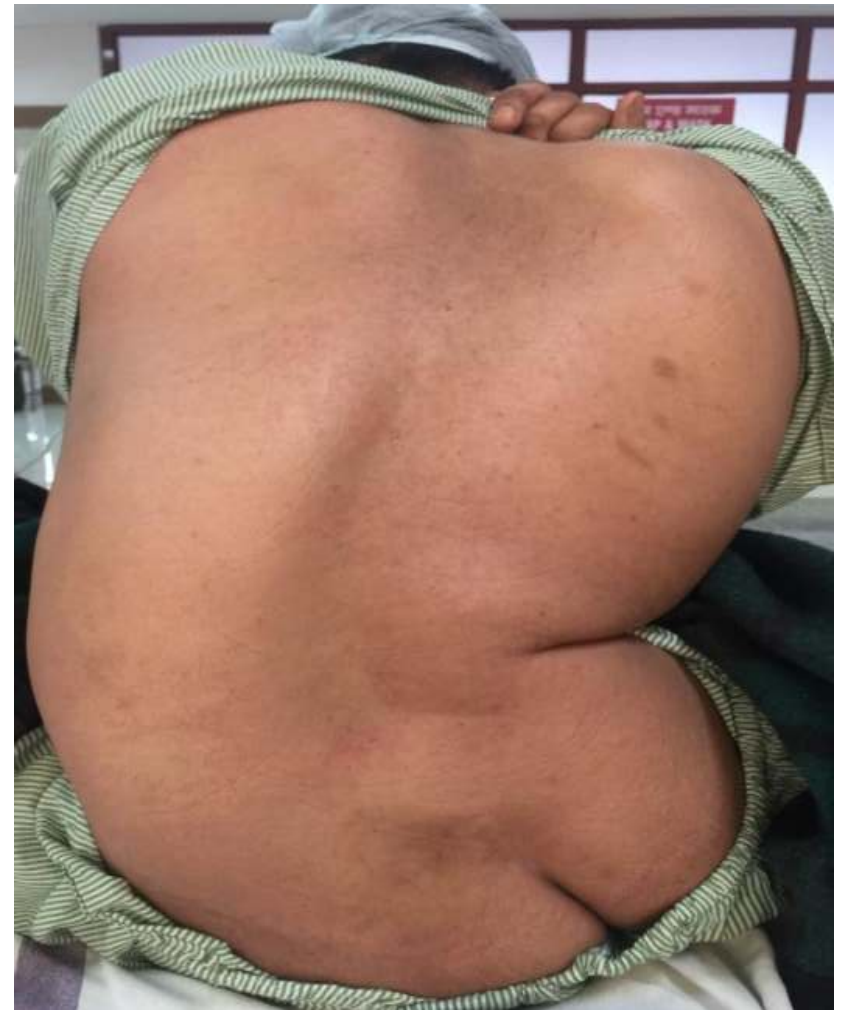

Fig a. Patient Spine

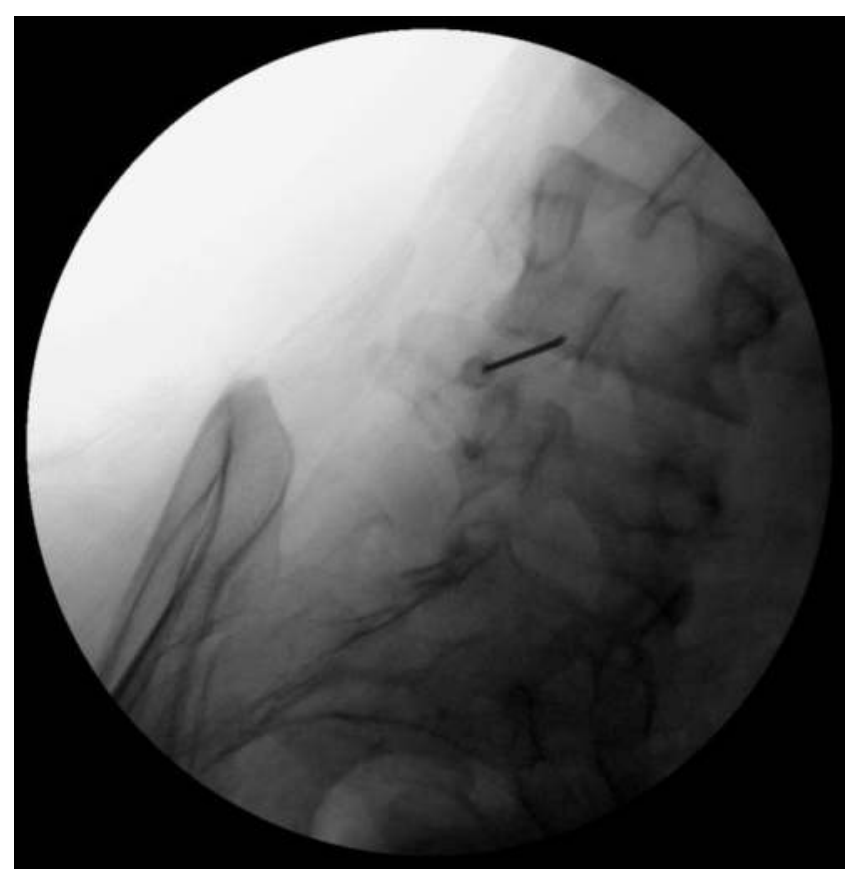

Fig c. Fluoroscopic anteroposterior view

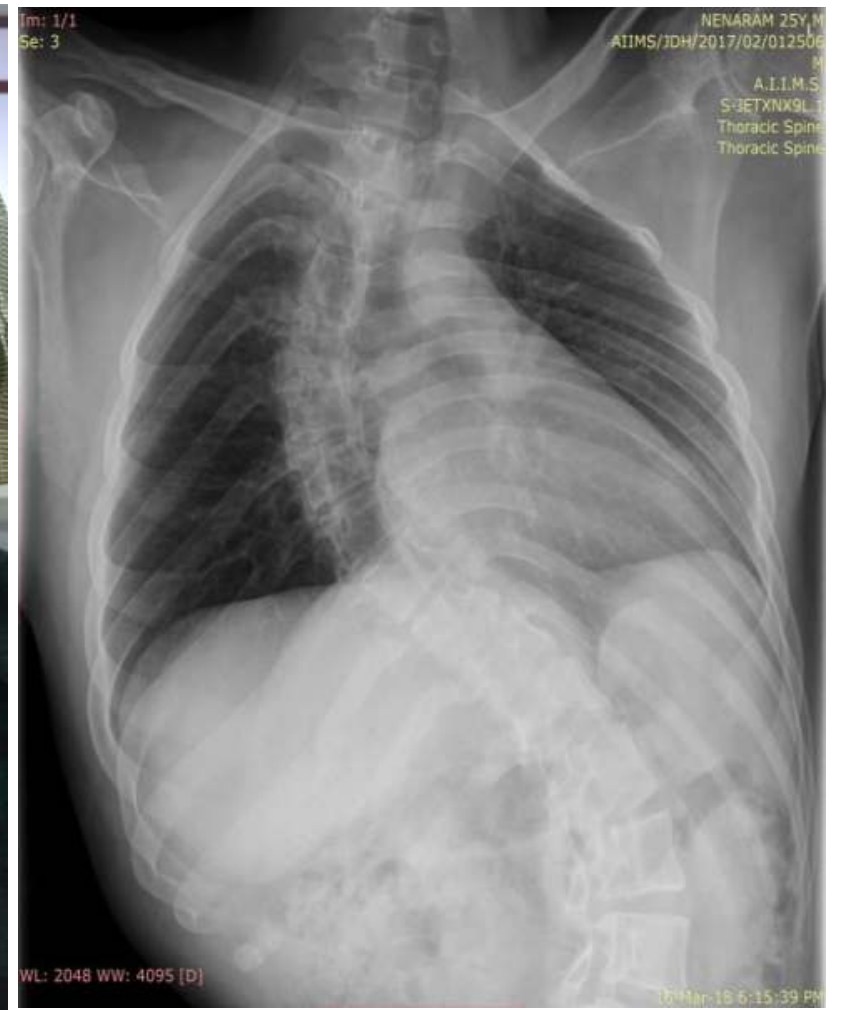

Fig b. X-ray spine

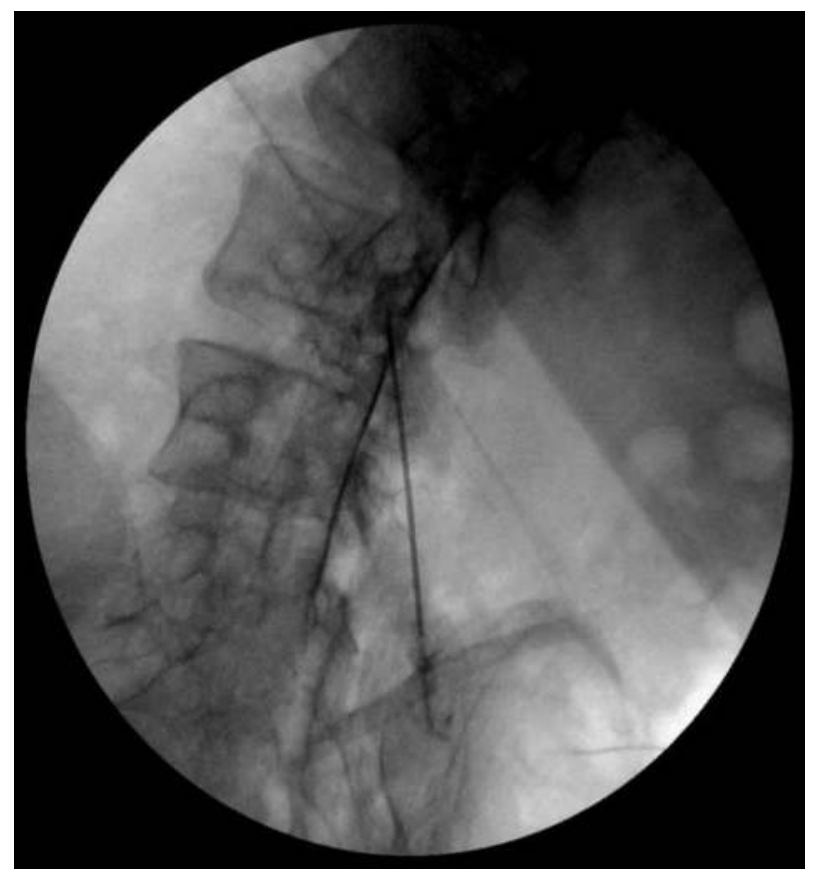

Fig d. Fluoroscopic lateral view with dye confirmation

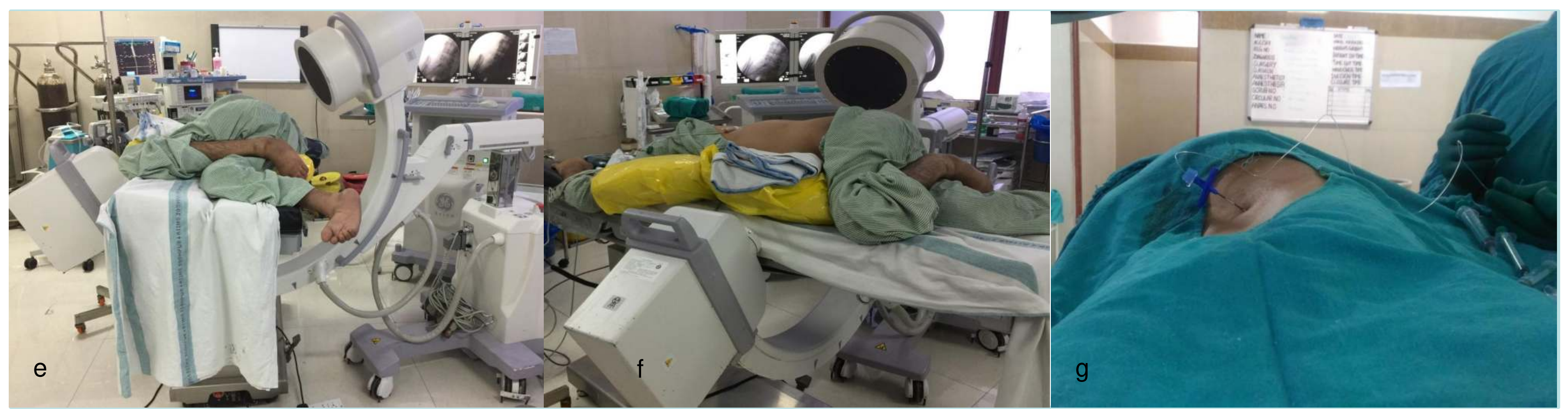

Fig e-g. Patient positioning and epidural needle in situ. 